\title{
Comparación fisicoquímica y biológica entre efluentes de la producción de trucha arcoíris y la condición limnológica del Lago Guamuez, departamento de Nariño
}

\author{
Physicochemical and biological comparison between effluents from \\ rainbow trout production and the limnological condition of Lake \\ Guamuez, department of Nariño
}

\section{Comparação físico-química e biológica entre efluentes da produção de trutas arco-íris e as condições limnológicas do lago Guamuez, departamento de Nariño}

Jorge N López-Macías ${ }^{1 *}$; Julbrinner Salas-Benavides ${ }^{2 *}$

1 MVZ, MSc, PhD;

2 Biol, MSc, (c)PhD

* Departamento de Recursos Hidrobiológicos; Facultad de Ciencias Pecuarias, Universidad de Nariño, Pasto, Colombia. Email: jorgelopezmacias@gmail.com

\begin{abstract}
Resumen
El estudio de lagos como fuentes de recursos naturales de diversa índole constituye una preocupación de entidades que regulan su manejo en conservación y producción sostenible en interacción de factores bióticos y abióticos que afectan el ecosistema. En consecuencia, éste proyecto puede generar información científica permanente para un plan de ordenamiento de la cuenca del lago Guamuez en cumplimiento de compromisos internacionales y nacionales en la conservación del humedal La Cocha. La presente investigación mediante 12 muestreos periódicos en cinco estaciones: 1. Intiyaco (E1), zona superficial $(A)$ y zona profunda $(B)$; las siguientes estaciones se ubicaron a partir de E1; estación 2. 50 metros $(C)$; 3. 100 metros $(D)$; 4 . 150 metros $(E)$ y 5 . Centro del lago $(F)$, análisis de varianza, prueba de normalidad y correlación, pretendió evaluar el efecto de las explotaciones acuícolas en jaulas flotantes sobre las variables fisicoquímicas y biológicas del lago Guamuez. La condición limnológica, presentó diferencias significativas entre aguas superficiales y profundas (respectivamente NH-N y DB, con P-PO, DQ, Colift y Coliff); el pH y NTU en A y B, difieren de los demás sitios evaluados. Existe mayor concentración $(P<0,05)$ de OD y \%O en estación 3. En el año 2008 las estaciones 1 y 5 señalan mayor media en la
\end{abstract}


estimación de OD, pH, Dur y DB y para el año 2018, aumentó los parámetros limitantes hacia el centro del lago (NH-N, P-PO, NTU y Coliff); así, el 70\% de los parámetros de evaluación en el proyecto Intiyaco, aumentaron su estimación media entre los años 2008 y 2018 (DB, OD y \%O). La concentración de $E$. coli presenta mayor media $(P<0,05)$ en B y difiere de $A, C, D, E$ y F; mientras fosfatos y Colift difieren $(P<0,05)$ entre $A$ y las estaciones $B, C, D, E$ y $F$.

Palabras clave: productividad primaria; coliformes; calidad de agua; deterioro ambiental

\begin{abstract}
The study of lakes as sources of natural resources of various kinds constitutes a concern of entities that regulate their management in conservation and sustainable production in interaction of biotic and abiotic factors that affect the ecosystem. Consequently, this project can generate permanent scientific information for a management plan for the Guamuez Lake basin in compliance with international and national commitments in the conservation of the La Cocha wetland. The present investigation by means of 12 periodic sampling in five stations: 1. Intiyaco (E1), superficial zone (A) and deep zone (B); the following stations were located from E1; station 2. 50 meters (C); 3. 100 meters (D); 4. 150 meters (E) and 5. Center of the lake $(\mathrm{F})$, analysis of variance, normality test and correlation, sought to evaluate the effect of aquaculture farms in floating cages on the physicochemical and biological variables of Lake Guamuez. The limnological condition showed significant differences between surface and deep waters (respectively $\mathrm{NH}-\mathrm{N}$ and $\mathrm{DB}$, with P-PO, DQ, Colift and Coliff); the $\mathrm{pH}$ and NTU in $A$ and $B$, differ from the other sites evaluated. There is a higher concentration $(P<0,05)$ of OD and \%O in station 3. In 2008, stations 1 and 5 show a higher average in the estimation of OD, pH, Dur and DB and for 2018, the parameters increased limiting towards the center of the lake (NH-N, P-PO, NTU and Colift); Thus, $70 \%$ of the evaluation parameters in the Intiyaco project increased their average estimate between 2008 and 2018 (DB, OD and \%O). The concentration of $E$. coli has a higher mean $(P<0,05)$ in $B$ and differs from $A, C, D$, E and F; while phosphates and Colift differ $(P<0,05)$ between $A$ and stations $B, C, D, E$ and $F$.
\end{abstract}

Key words: primary productivity; coliforms; water quality; environmental deterioration

\begin{abstract}
Resumo
O estudo de lagos como fontes de recursos naturais de vários tipos constitui uma preocupação de entidades que regulam seu manejo na conservação e produção sustentável na interação de fatores bióticos e abióticos que afetam o ecossistema. Consequentemente, este projeto pode gerar informações científicas permanentes para um plano de manejo da bacia do lago Guamuez, em conformidade com os compromissos internacionais e nacionais na conservação do pantanal de La Cocha. A presente investigação por meio de 12 amostragens periódicas em cinco estações: 1. Intiyaco (E1), zona superficial (A) e zona profunda (B); as seguintes estações foram localizadas a partir da E1; estação 2. 50 metros (C); 3.100 metros (D); 4. 150 metros (E) e 5. Centro do lago (F), análise de variância, teste de normalidade e correlação, procuraram avaliar o efeito de fazendas de aquicultura em gaiolas flutuantes nas variáveis físico-químicas e biológicas do lago Guamuez. A condição limnológica mostrou diferenças significativas entre águas superficiais e profundas (respectivamente $\mathrm{NH}-\mathrm{N}$ e DB, com P-PO, DQ, Coliff e Coliff); O pH e NTU em A e B diferem dos demais locais avaliados. Há uma maior concentração $(\mathrm{P}<0,05)$ de $\mathrm{DO}$ e $\% \mathrm{O}$ na estação 3. Em 2008, as estações 1 e 5 apresentam uma média mais alta na estimativa de DO, pH, Dur e DB e, para 2018, os parâmetros aumentaram limitando em direção ao centro do lago (NH-N, P-PO, NTU e Colift $)$; Assim, 70\% dos parâmetros de avaliação no projeto Intiyaco aumentaram sua estimativa média entre 2008 e 2018 (DB, OD e \%O). A concentração de E. coli tem uma média mais alta $(\mathrm{P}<0,05)$ em $\mathrm{B}$ e difere de $\mathrm{A}, \mathrm{C}, \mathrm{D}$, $\mathrm{E}$ e $\mathrm{F}$; enquanto os fosfatos e Colift diferem $(P<0,05)$ entre $A$ e as estações $B, C, D, E$ e $F$.
\end{abstract}

Palavras chave: produtividade primária; coliformes; qualidade da agua; deterioração ambiental

\section{Introducción}

La población mundial, aún en países en desarrollo, está sufriendo un rápido cambio hacia la urbanización, lo que hace que el hombre haya llegado a ser más dependiente del suministro comercial de alimento a través de mercados organizados, que intervengan en la cadena de seguridad alimentaria. Lo anterior, incrementa la presión sobre los recursos naturales, sobretodo en aguas continentales y marinas; los cuales son altamente susceptibles a la contaminación y que han obligado a las pesquerías naturales a disminuir, pese a su gran potencial aún inexplotado y que han desempeñado un papel importante en la generación, no sólo de alimentos, sino también de divisas. El deterioro del medioambiente y especialmente en el lago Guamuez, es causado principalmente por las descargas de basuras y aguas residuales domésticas, provenientes de la población El Encano. La deforestación intensa de los bosques naturales de la cuenca y la sustitución de estos por actividades agrícolas y ganaderas que erosionan los suelos e incrementan la cantidad de sólidos disueltos en el lago Guamuez, deteriora el hábitat de los peces, dificulta la alimentación de 
especies ícticas bentónicas, aumentan la turbidea (NTU) y disminuyen el oxígeno disuelto, lo cual afecta mecánicamente los procesos de respiración, visión y olfato de los peces y por ende, la respuesta inmunológica, haciéndolos más susceptibles a la ruptura de la relación mutual con los parásitos, los cuales causan la enfermedad. (Gilmore et al., (2010); Rábago-Castro (2010); Rubio-Godoy et al., (2012). De esta manera, la truchicultura, frente a formas de producción como la agricultura y agroforestería, incentiva el manejo adecuado de las fuentes de agua, la conservación de los bosques primarios, el manejo adecuado de las cuencas hidrográficas, uso integral y el manejo racional de las materias primas y recursos locales, aumenta la productividad, reduce los costos unitarios de producción e incorpora valor agregado. Así mismo, la acuicultura, adquirió gran importancia dentro del sistema alimentario por la producción de bienes de gran relevancia desde el punto de vista nutricional y alcanzar áreas deprimidas o de mediano desarrollo, donde puede originar una serie de actividades conexas como la pesca deportiva y el turismo ecológico que estimulan el crecimiento económico. Los cultivos piscícolas, aprovechan económicamente el agua para la producción de proteína animal en sistemas de alta densidad en volúmenes pequeños; condiciones que favorecen la propagación de enfermedades por la acumulación de metabolitos y deterioro de condiciones fisicoquímicas (oxígeno, solubilización y/o precipitación de materia orgánica, pH, temperatura) y bacteriológicas del medio acuático, generando grandes pérdidas económicas cuando alteran las variables de incremento de peso, talla, conversión alimenticia y sobrevivencia que justifica tomar medidas para prevenir esos detrimentos. La presencia de agentes patógenos y huéspedes apropiados favorecen la aparición de enfermedades, frente a lo cual el piscicultor utiliza gran cantidad de medicamentos y químicos (representan hasta el 10\% de los costos), generando, no sólo resistencia de los microorganismos, sino también la destrucción del fitoplancton y por ende, la alteración total de la productividad primaria del medio acuático (López et al., (2005); López (2014). Acorde a lo anterior, esta investigación realizó un diagnóstico del estado fisicoquímico y biológico de las descargas provenientes de la producción de trucha arcoíris (Oncorhynchus mykiss) de la estación piscícola Intiyaco en el lago Guamuez, sobre el mismo sistema, con el fin de establecer la correlación que existe entre estos parámetros y la biota nativa y así definir su papel limitante en la condición limnológica la- custre y afianzar una política de intervención sobre los sistemas ícticos productivos efluentes. Los resultados contribuirán a establecer las condiciones favorables en la productividad natural del sistema lacustre y a fortalecer el rendimiento somático que requiere las empresas piscícolas de la región cuyo establecimiento ha representado para sus moradores seguridad alimentaria sin detrimento de la relación enteramente dinámica: el hombre, las demás especies y el hábitat que define un sistema ecológico.

\section{Materiales y métodos}

\section{Localización}

El presente estudio se realizó en el Lago Guamuez, corregimiento El Encano, ubicado a 25 kilómetros al oriente la ciudad de San Juan de Pasto, departamento de Nariño; coordenadas geográficas $1^{\circ} 06^{\prime} 42,53^{\prime \prime}$ latitud Norte y $77^{\circ} 08^{\prime} 29,15^{\prime \prime}$ longitud Oeste, altura en cota máxima y mínima 2,850 msnm y 2,745 msnm; temperatura ambiental media $11,6^{\circ} \mathrm{C}$ (variaciones 6 y $14^{\circ} \mathrm{C}$ ), humedad relativa $86 \%$, brillo solar 898,2 horas anuales y precipitación pluvial 1.371 $\mathrm{mm}$ anuales; volumen aproximado $1700 \mathrm{hm}^{3}$ y tiempo de residencia estimado en 6,22 años. (CORPONARIÑO 2014).

\section{Análisis físico y químico del sistema lenítico}

La investigación alcanzó doce muestreos (bimestral), en cinco sitios (tres réplicas); cuatro de ellos, fueron puntos del lago seleccionados de acuerdo a la dirección del viento vía centro del sistema lacustre, ubicados cada 50 m después de la estación Piscícola Intiyaco (E1), hasta 150 (E) y finalmente centro del lago (F) a 450 m, designada como control, merced al deslindamiento de la producción acuícola autorizada por Corpanariño, realizados durante 24 meses abarcando las épocas de máxima y mínima precipitación, seleccionados para la estimación física, química y microbiológica (Colift y Coliff). El disco Secchi (Secchi PA 1865), se utilizó para medir la transparencia Secchi (ZSD), que define la Zona Fótica y Afótica expresada en metros; la Profundidad de la Zona Fotica se consiguió multiplicando ZSD por la constante de Pool y Atkins $(2,7)$.

Los parámetros estimados y analizados en mg/l, correspondieron a Oxígeno disuelto (OD), saturación de oxígeno (\%O), $\mathrm{DBO}_{5}(\mathrm{DB}), \mathrm{DQO}(\mathrm{DQ}), \mathrm{CO}_{2}(\mathrm{CO})$, Alcalinidad (Alc), NTU, Dureza (Dur), Nitrógeno amoniacal $(\mathrm{NH}-\mathrm{N})$ y Fosfatos (P-PO); y con sus pro- 
pias unidades la Temperatura $\left(\mathrm{T}\right.$ en $\left.{ }^{\circ} \mathrm{C}\right), \mathrm{pH}\left[\mathrm{H}^{+}\right]$, Zonas Fótica - Afótica (m) y Coliformes fecales (Coliff) y Coliformes totales (Colif $f_{t}$ en UFC.

La caracterización fisicoquímica se realizó en las aguas superficiales y profundas (varía de acuerdo al régimen pluviométrico) de los sitios de muestreo; para tal efecto, se tomó $2 \mathrm{~L}$ de muestras con botella tipo Van Dorn, un litro se destinó al análisis fisicoquímico y el otro en la caracterización biológica. In situ se estimó mediante equipo electrométrico, los parámetros: T, OD, CO, pH, Alc, NTU y Dur; Zonas Fótica y Afótica (Hela y Laveastu, (1961)); los demás parámetros químicos ya descritos, se estimaron por Standard Methods for the Examination of Water and Wastewater en los Laboratorios Especializados y Laboratorio de Calidad de Aguas para Acuacultura del Programa de Ingeniería Acuícola de la Universidad de Nariño.

\section{Análisis estadístico}

La investigación es cuantitativa y por el objetivo es descriptiva, explicativa y longitudinal, aplicación de prueba de hipótesis (Comparativa) y correlaciones para definir o determinar relación causal o de dependencia entre variables abióticas (fisicoquímicas) y bióticas (Colift y Coliff), nivel de significancia $\alpha=0,05$ (5\%), procesadas en el paquete estadístico IBM SPSS Statistics 20.

\section{Resultados}

\section{Condición física y química}

El análisis descriptivo de la condición fisicoquímica y biológica del lago Guamuez en correlación con la Estación Piscícola Intiyaco, permite definir que la media de compuestos altamente eutroficantes como P-PO y $\mathrm{NH}-\mathrm{N}$, el coeficiente de relación se estableció en 1:54, significando entonces que por cada mg/L de P-PO, la concentración NH-H seria 54 $\mathrm{mg} / \mathrm{L}$; este mismo análisis entre DB y DQ señaló un indicador de 1:5,6, y finalmente 1:16,9 para Coliff y Colift. (Tabla 1).

La condición física asociada a los efluentes truchícolas de la Estación Piscícola Intiyaco en relación con la absorción de la energía lumínica en el sistema Lago Guamuez permitieron inferir la existencia de diferencias significativas desde ésta producción que aporta material particulado de origen fecal y alimentos, los cuales se van disipando hasta el centro del lago, mejorando así su penetrabilidad (33,5 m). (Tabla 1).

De otro lado, la caracterización química del sistema Estación Piscícola Intiyaco en comparación con el control $(E)$, definió diferencias significativas $(P<0,05)$; se destaca la mayor media en aguas superficiales sobre aguas profundas en los parámetros NTU, NH-N y mejor media de aguas profundas sobre aguas superficiales y el centro del lago en la condición de Coliff, Colift y

Tabla 1. Anova. Comparaciones múltiples. Condición fisicoquímica y biológica. Lago Guamuez - Estación Piscícola Intiyaco.

\begin{tabular}{|l|r|r|r|r|r|r|}
\hline \multicolumn{1}{|c|}{ Parámetro } & A & B & C & D & E & F \\
\hline pH & $7,1^{\mathrm{a}}$ & $71^{\mathrm{a}}$ & $7,0^{\mathrm{b}}$ & $6,7^{\mathrm{c}}$ & $6,8^{\mathrm{d}}$ & $6,6^{\mathrm{e}}$ \\
\hline NTU mg/l & $198,9^{\mathrm{a}}$ & $198,3^{\mathrm{b}}$ & $198,0^{\mathrm{c}}$ & $197,6^{\mathrm{d}}$ & $197,2^{\mathrm{e}}$ & $41,7^{\mathrm{f}}$ \\
\hline Zona Fótica m & $4,2^{\mathrm{f}} \mathrm{m}^{\mathrm{f}}$ & $7,50 \mathrm{~m}^{\mathrm{e}}$ & $16,7^{\mathrm{d}}$ & $23,8 \mathrm{~m}^{\mathrm{c}}$ & $30,0 \mathrm{~m}^{\mathrm{b}}$ & $33,5^{\mathrm{a}}$ \\
\hline OD mg/l & $5,7^{\mathrm{e}}$ & $5,65^{\mathrm{e}}$ & $5,8^{\mathrm{d}}$ & $5,87^{\mathrm{c}}$ & $5,93^{\mathrm{b}}$ & $6,53^{\mathrm{a}}$ \\
\hline Dur mg/l & $23,3^{\mathrm{e}}$ & $23,0^{\mathrm{e}}$ & $30,3^{\mathrm{a}}$ & $29,3^{\mathrm{b}}$ & $28,0^{\mathrm{c}}$ & $26,1^{\mathrm{d}}$ \\
\hline Alc mg/l & $15,6^{\mathrm{e}}$ & $15,4^{\mathrm{e}}$ & $23,4^{\mathrm{a}}$ & $20,7^{\mathrm{b}}$ & $18,7^{\mathrm{c}}$ & $17,6^{\mathrm{d}}$ \\
\hline NH-N mg/l & $3,23^{\mathrm{a}}$ & $3,12^{\mathrm{b}}$ & $3,10^{\mathrm{c}}$ & $3,10^{\mathrm{c}}$ & $3,10^{\mathrm{c}}$ & $3,00^{\mathrm{d}}$ \\
\hline Coliff UFC & $55,043^{\mathrm{b}}$ & $100^{\mathrm{a}}$ & $55,5^{\mathrm{b}}$ & $53,3^{\mathrm{b}}$ & $51,6^{\mathrm{b}}$ & $49,9^{\mathrm{b}}$ \\
\hline Colift UFC & $931,043^{\mathrm{b}}$ & $1466,7^{\mathrm{a}}$ & $900,076^{\mathrm{b}}$ & $837,34^{\mathrm{b}}$ & $789,89^{\mathrm{b}}$ & $750,38^{\mathrm{b}}$ \\
\hline P-PO mg/l & $0,060^{\mathrm{b}}$ & $0,2250^{\mathrm{a}}$ & $0,0193^{\mathrm{c}}$ & $0,0186^{\mathrm{c}}$ & $0,0175^{\mathrm{c}}$ & $0,0164^{\mathrm{c}}$ \\
\hline
\end{tabular}

a, b, c, d, e, f. significación estadística. 
Tabla 2. Anova. Comparaciones múltiples. Condición fisicoquímica y biológica. Aguas superficiales y profundas Estación Intiyaco.

\begin{tabular}{|l|c|c|}
\hline \multicolumn{1}{|c|}{ Parámetro } & $\begin{array}{c}\text { A } \\
\text { (Superficiales) }\end{array}$ & $\begin{array}{c}\text { B } \\
\text { (Profundidad) }\end{array}$ \\
\hline $\mathrm{pH}$ & $7,1^{\mathrm{a}}$ & $7,1^{\mathrm{a}}$ \\
\hline $\mathrm{NTU} \mathrm{mg} / \mathrm{l}$ & $198,9^{\mathrm{a}}$ & $198,7^{\mathrm{a}}$ \\
\hline Zona Fótica m & $4,2 \mathrm{~m}^{\mathrm{a}}$ & $7,50 \mathrm{~m}^{\mathrm{b}}$ \\
\hline OD mg/l & $5,7^{\mathrm{a}}$ & $5,65^{\mathrm{a}}$ \\
\hline Dur mg/l & $23,3^{\mathrm{a}}$ & $23,0^{\mathrm{a}}$ \\
\hline Alc mg/l & $15,6^{\mathrm{a}}$ & $15,4^{\mathrm{a}}$ \\
\hline P-PO mg/l & $0,060^{\mathrm{b}}$ & $0,2250^{\mathrm{a}}$ \\
\hline NH-H mg/l & $3,23^{\mathrm{a}}$ & $3,12^{\mathrm{b}}$ \\
\hline DB mg/l & $4,0^{\mathrm{a}}$ & $2,29^{\mathrm{b}}$ \\
\hline DQ mg/l & $22,57^{\mathrm{b}}$ & $27,435^{\mathrm{a}}$ \\
\hline Colift UFC/100 $\mathrm{mL}^{-1}$ & $931,043^{\mathrm{b}}$ & $1466,7^{\mathrm{a}}$ \\
\hline Coliff UFC/100 $\mathrm{mL}^{-1}$ & $55,043^{\mathrm{b}}$ & $100^{\mathrm{a}}$ \\
\hline
\end{tabular}

a, b. significación estadística.

P-PO, que presento mayor media en la variable OD. (Tabla 1).

La presencia de Coliff y Colift, entre las estaciones permitió determinar diferencias significativas $(\mathrm{P}<0,05)$ en esta condición biótica referida al sistema Lago Guamuez y las aguas superficiales y profundas de la
Estación Intiyaco. El estudio develó mayor media de Coliff y Colift en aguas profundas que supera respectivamente en 57,5 y $81,6 \%$ en relación a las aguas superficiales. (Tabla 2).

La prueba de significancia estadística, aplicada para la condición limnológica del Lago Guamuez incluido los efluentes de la producción piscícola Intiyaco, presentó diferencias significativas $(\mathrm{P}<0,05)$ en $\mathrm{pH}(\overline{\mathrm{X}}=7,1)$ y NTU $(\bar{X}=198,9)$ en las zonas de muestreo C, D, E y F, y no así en aguas superficiales y profundas del sistema truchícola de evaluación; mientras que los parámetros OD $(\overline{\mathbf{X}}=5,7)$, Dur $(\overline{\mathbf{X}}=23,3)$ y Alc $(\overline{\mathbf{X}}=15,6)$ aparecen con menor media en estas aguas. La concentración de $\mathrm{NH}-\mathrm{N}(\overline{\mathbf{X}}=0,4462)$ presenta mayor media en aguas superficiales y disminuye su concentración por litro en la medida que las aguas se distancian del proyecto de Intiyaco (A, C, D, E y F; por su parte, P-PO ( $\bar{X}=0,2250)$ con mayor media en aguas superficiales del sistema de producción de O. mykiss, se reducen hacia el fondo de la misma y una vez las aguas se evalúan después de Intiyaco hacia el centro del lago (B, C, D, E y F), como aparece en la Figura 1.

\section{Comunidad microbiológica}

La comunidad microbiana del Lago Guamuez inferida desde los componentes enterobacteriales coliformes durante el periodo de evaluación, permite definir que Colift cronológicamente presentaron diferencias significativas en los puntos distantes a la producción piscícola. La prueba ANOVA ha definido diferencias altamente significativas en las condiciones

Tabla 3. Matriz de correlaciones fisicoquímicas. Lago Guamuez - Estación Piscícola Intiyaco.

\begin{tabular}{|l|l|c|c|c|c|c|c|}
\hline \multicolumn{2}{|c|}{ Parámetros } & P-PO & NH-N & DB & DO & Colift & Coliff \\
\hline \multirow{2}{*}{ P-PO } & Correlación de Pearson & $\mathbf{1}$ & 0,467 & $-0,013$ & $-0,138$ & 0,379 & $-0,540^{*}$ \\
\cline { 2 - 8 } & Sig. (bilateral) & & 0,068 & 0,961 & 0,609 & 0,147 & 0,031 \\
\hline \multirow{2}{*}{ NH-N } & Correlación de Pearson & 0,467 & $\mathbf{1}$ & 0,176 & $-0,113$ & $0,698^{* *}$ & $-0,198$ \\
\cline { 2 - 8 } & Sig. (bilateral) & 0,068 & & 0,514 & 0,677 & 0,003 & 0,463 \\
\hline \multirow{2}{*}{ DB } & Correlación de Pearson & $-0,013$ & 0,176 & $\mathbf{1}$ & $-0,745^{* *}$ & 0,238 & $-0,135$ \\
\cline { 2 - 8 } & Sig. (bilateral) & 0,961 & 0,514 & & 0,001 & 0,374 & 0,618 \\
\hline \multirow{2}{*}{ DQ } & Correlación de Pearson & $-0,138$ & $-0,113$ & $-0,745^{* *}$ & $\mathbf{1}$ & $-0,266$ & 0,259 \\
\cline { 2 - 8 } & Sig. (bilateral) & 0,609 & 0,677 & 0,001 & & 0,319 & 0,333 \\
\hline \multirow{2}{*}{ Coliff } & Correlación de Pearson & 0,379 & $0,698^{* *}$ & 0,238 & $-0,266$ & $\mathbf{1}$ & $-0,754^{* *}$ \\
\cline { 2 - 8 } & Sig. (bilateral) & 0,147 & 0,003 & 0,374 & 0,319 & & 0,001 \\
\hline \multirow{2}{*}{ Coliff } & Correlación de Pearson & $-0,540^{*}$ & $-0,198$ & $-0,135$ & 0,259 & $-0,754^{* *}$ & $\mathbf{1}$ \\
\cline { 2 - 8 } & Sig. (bilateral) & 0,031 & 0,463 & 0,618 & 0,333 & 0,001 & \\
\hline
\end{tabular}

* La correlación es significativa en el nivel 0,05 (bilateral).

** La correlación es significativa en el nivel 0,01 (bilateral). 


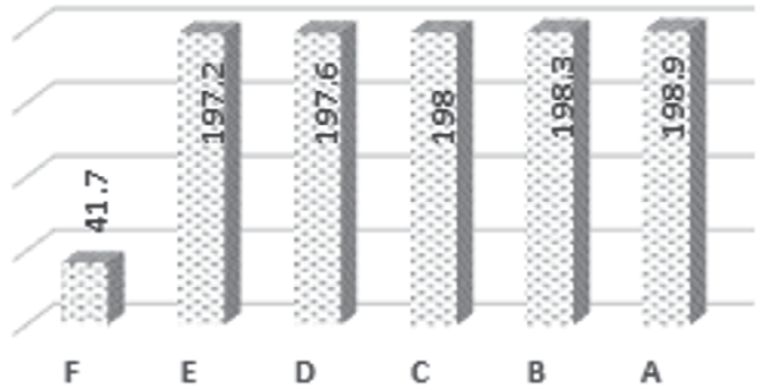

OD
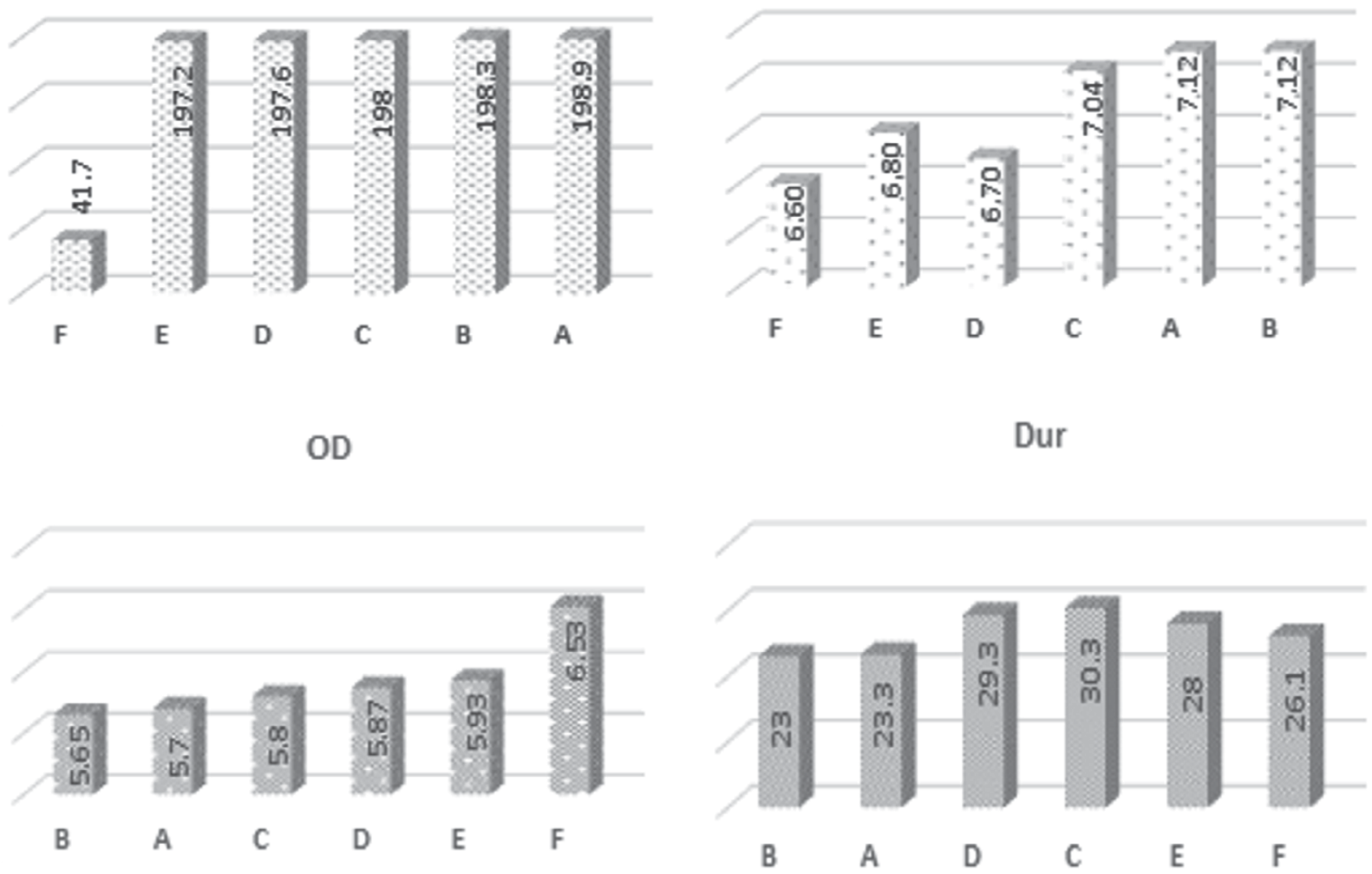

Alc

NH-N

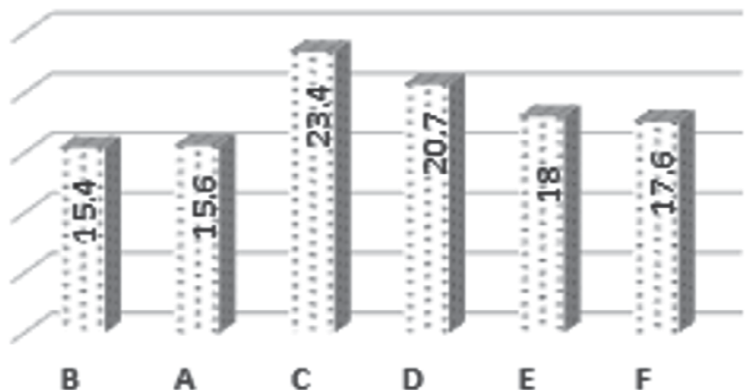

P-PO

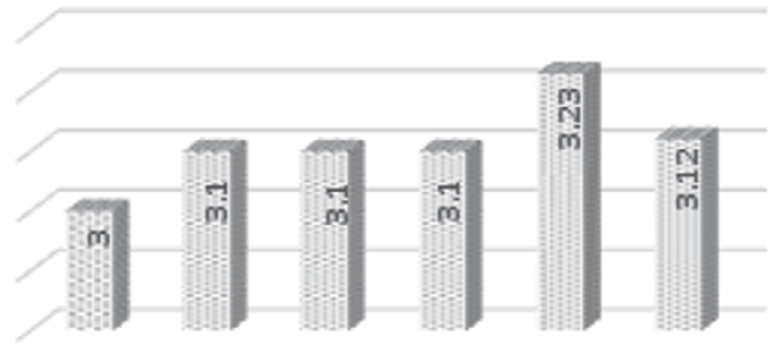

$$
\text { F }
$$

E

D

C

A B

ZSD
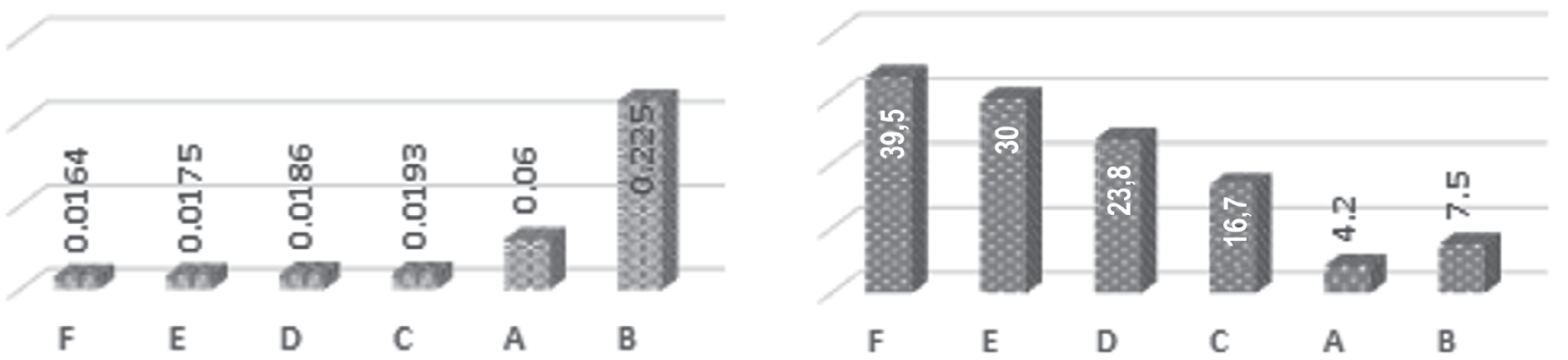

Figura 1. SPSS. Comparaciones múltiples. HSD Tukey a, b. Condición fisicoquímica Lago Guamuez - Estación Piscícola Intiyaco, a. Utiliza el tamaño de la muestra de la media armónica $=26,000$. b. Alfa $=0,05$. a, b, c, d, e = significación estadística. 


\section{Colif $_{f}$}

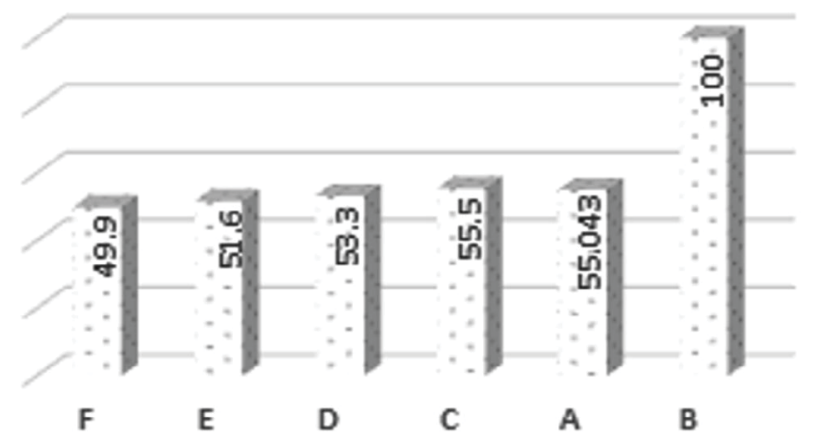

Colif $_{\mathrm{t}}$

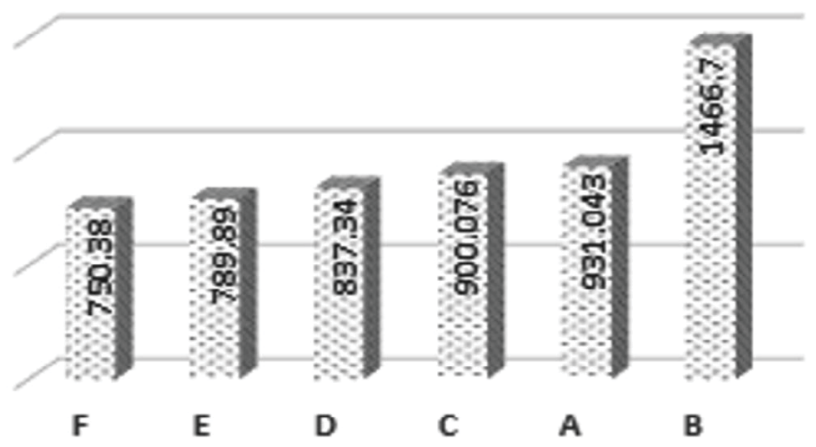

Figura 2. SPSS. Comparaciones múltiples. HSD Tukey a, b. Condición fisicoquímica Lago Guamuez - Estación Piscícola Intiyaco. a. Utiliza el tamaño de la muestra de la media armónica $=26,000$. b. Alfa $=0,05$. a, b, c, d, e = significación estadística.

de P-PO $(P<0,00)$, Colift $(P<0,020)$ y Coliff $(P<0,001)$. Existen diferencias en la concentración P-PO entre las aguas superficiales (media 0,2250) del sistema de producción y los demás puntos de muestreo. La mayor media $(1.466,7)$ de Colift está presente en aguas superficiales de la producción de O. mykiss y difiere significativamente de las profundas $(190,0)$. La prevalencia de E. coli en aguas profundas (media 100) difiere en concentración con los demás sitios de análisis. Los demás parámetros se mantuvieron dentro de la distribución normal, en consecuencia, no existe diferencias estadísticas significativas entre sus concentraciones. (Figura 2).

\section{Análisis de correlaciones fisicoquímicas y biológicas}

Mediante análisis de correlación de Pearson se identificó asociación lineal significativa entre la concentración de $\mathrm{NH}-\mathrm{H}$ y la prevalencia de Colift $(\mathrm{R}=0,698$; $\mathrm{P}<0,001)$; igualmente, asociación lineal negativa significativa entre la $D B$ y $D Q(R=-0,745 ; P<0,001)$. En consecuencia, según el coeficiente de determinación $\left(R^{2}=0,55\right.$ y 0,48$)$, se puede inferir que la variabilidad de $\mathrm{NH}-\mathrm{H}$ se explica en $55 \%$ por la variabilidad en la concentración de Colift; y en $48 \%$ se explica la variabilidad de la demanda biológica y química de las aguas del Lago Guamués. (Tabla 3).

\section{Discusión}

El estudio pretendió verificar el efecto de los efluentes de la producción de pescado en la calidad física, química y biológica natural del Lago Guamués, más no su consecuencia en las variables zootécnicas de esa actividad, y fue así como, la estimación de la zona trofogénica, que involucra las capas eufótica y fótica del sistema lenítico aumenta en la medida que se aleja de las estaciones de producción acuícola que limita la productividad primaria y la concentración de oxígeno al atenuar la actividad fotosintética por presencia de mayor turbidez relacionada al material disuelto y suspendido resultado de excretas, alimento y fluidos biológicos de la evisceración de animales, que determina mayor albedo (Kormondy (1994)). En correlación con la mayor concentración de oxígeno y saturación de este gas, presente significativamente a 100 metros a después de las jaulas de producción piscícola Intiyaco, refleja ese efecto, el cual se mitiga bajo las nuevas condiciones, la presencia de la comunidad fitoplanctónica, verificada como dominante, abundante y mayor riqueza de las Divisiones Bacillariophytas $(44.4 \%)$ Clorophytas $(37 \%)$ y Cianophytas $(18.6 \%)$, que conllevan relaciones energéticas a partir de la energía del flujo solar que se consume en el proceso de la fotosíntesis para sintetizar compuestos orgánicos carbonados (Carney (1984); Carney et al., (1987) y Kirk (1994)); además, prevalecen para dinamizar los niveles tróficos superiores definidos por las poblaciones Diaptomus sp y Peridinium $s p$ $(100 \%)$ y menos frecuentes las especies Bosmina $s p$, Paramecium sp, Metacyclops sp y Keratella $s p$ $(33,3 \%)$, en términos de transferencia de materia (ciclos biogeoquímicos), energía y biodisponibilidad (Zapata (2001); Granados et al., (2008), Andramunio-Acero y Caraballo (2012) y Andramunio-Acero (2013)); igualmente, estos recursos bióticos son considerados como indicadores de calidad ambiental debido a la sensibilidad o tolerancia a diversos factores abióticos que les asiste (Valdés y Plaza (2011); Roldán (2003)).

La condición NH-N y DB presentan diferencias significativas con mayor media en las aguas superficiales, 
auspiciada por la sobresaturación proveniente de los procesos de alimentación-ingestión, egestión, y la utilización del OD por el plancton en la actividad respiratoria y en la descomposición de la materia orgánica acumulada en la superficie, en concomitancia con el fondo de la columna de agua que no supera los 17 metros en los sitios evaluados (Sánchez y Zea (2000); Granados et al., (2008), López y Salas (2013)). Adicionalmente, el aprovisionamiento en nutrientes en un lago quedará afectado por la biogeoquímica de la cuenca de drenaje, por los contaminantes de origen ribereño y atmosférico y por los procesos lacustres (Vincent et al., (1984)).

El comportamiento del P-PO, DQ y Colift es significativamente diferencial con mayor media en aguas profundas, como consecuencia de la presencia del fosforo como elemento limitante que facilita la productividad primaria (Smith (1983); Elser et al., (1990); Sánchez y Zea (2000)), sin embargo, en aguas profundas puede causar afloramientos excesivos que causan sofocamiento de todos los organismos vivientes por bajas drásticas de oxígeno útil en la mineralización de la materia orgánica (Khalida et al., 2011) de origen autóctona y alóctona, produciendo la muerte cuando facilita los procesos de sedimentación, mineralización y eutrofización litoral (Cabrera et al., (2005); Ding et al., (2010), López y Salas (2013)).

Las diferencias significativas entre zonas de estudio (C, D, E y F), en la condición de pH, NTU (mayor media), OD, Dur y Alc (menor media) del sistema productivo Intiyaco, se correlaciona con la materia inorgánica, material orgánico particulado, dinámica hídrica, densidad del agua y frecuencia de mezcla que afectan la penetración lumínica asociada a NTU (media 198.9) y la condición de pH donde prevalece los bicarbonatos y menor concentración de carbonatos y dióxido de carbono relacionado a los niveles Alc total $(\overline{\mathbf{X}}=15,5)$ y Dur $(\overline{\mathbf{X}}=23,2)$, en concordancia con la insuficiencia de calcio para la coprecipitación del fosfato cálcico con el carbonato de calcio como mecanismo de autopurificación de fosfato más eficiente, así como concentración baja de OD $(\overline{\mathbf{X}}=5,7)$, merced a los procesos de oxidación de la materia disponible (Roldán (1992); Roldán y Posada (2001); López y Salas (2013)).

El NH-N y la concentración de E. coli presentaron mayor media en aguas profundas y difiere de las zonas $(A, C, D, E$ y $F)$; si bien, se ha demostrado que en lagos de las regiones tropicales, la densidad fitoplactónica está más frecuentemente limitados por el nitrógeno que por el fósforo (Vincent et al., (1984)), sin embargo, éste comportamiento obedece a la decantación de las fuentes de nitrógeno, relacionada con el posafloramiento de productores primarios y su mineralización con presencia de Colift y prevalencia de Coliff por cercanía a los fondos desde los vertimientos superficiales (55 UFC).

Los P-PO y Colift de aguas profundas son significativamente diferentes de aguas superficiales de la producción de O. mykiss y los demás sitios de evaluación (B, C, D, E y F) en correspondencia a la decantación de alimento no consumido, heces fecales y mineralización planctónica (Sánchez de Prager et al., (2006); Ordoñez et al., (2016) y Varma (2017)), metabolismo de macromoléculas vía procesos de hidrólisis, acetogénesis y metanogénesis entre otros (Corrales et al., 2015).

Según la Corporación autónoma regional de Nariño, de acuerdo al modelo dinámico de capacidad de carga (MDCC), incluido los peces cultivados y los nativos Eremophilus mutissi y Grundulus bogotensis. (CORPONARIÑO, Resolución 1006 de 2015), la carga de nutrientes, específicamente de nitrógeno y fosforó, prevé una capacidad permanente máxima de sostenimiento del lago igual a 930 ton/año; que representa una capacidad de carga muy limitada debido a la condición de lago de altiplano, oligotrófico con escasa tensión superficial de OD y mayor aporte de M.O por aguas vertidas de diferentes asentamientos, de áreas deforestadas y zonas de explotación ganadera, actividades agrícolas en la microcuenca, las cuales transportan pesticidas y fertilizantes, contaminación atmosférica y los procesos propio lacustres que presumiblemente podrían causar serios problemas de eutrofización.

En conclusión, el análisis de la estación de interés y el sitio control durante el año 2018, los parámetros limitantes en la condición limnológica del lago están en la concentración de NH-H, P-PO, NTU y Colift que descienden en dirección hacia el centro del Lago Guamuez, sin embargo, no se evidenció un deterioro negativo, en la caracterización fisicoquímica y biológica, causado por las actividades acuícolas, considerando la capacidad de autopurificación y la condición polimíctica temporal del sistema lenítico, sin embargo, se debe continuar con los procesos de mejoramiento de técnicas de elaboración de alimentos de alta digestibilidad y estrategias de alimentación que mitigue las condiciones de mayor concentración de nitrógeno amoniacal, turbidez y pH, que en lo pertinente limitan la productividad primaria con la consecuente declinación de la concentración y saturación de oxígeno en las explotaciones piscícolas. 


\section{Agradecimientos}

El grupo de investigaciones acuícolas (GIAC), expresan sincera gratitud a las personas e instituciones que hicieron posible el desarrollo de la investigación "Impacto aeróbico y anaeróbico de la especie exótica trucha arcoíris (Oncorhynchus mykiss), en un lago altoandino colombiano"

Personal Administrativo y Profesional de la Vicerrectoría de Investigaciones Postgrados y Relaciones Internacionales de la Universidad de Nariño.

Personal Administrativo y Profesional del Departamento de Recursos Hidrobiológicos de la Universidad de Nariño.

Personal Administrativo y Profesional de la Sección de Laboratorios de la Universidad de Nariño.

\section{Referencias}

Andramunio-Acero C, Caraballo P. Análisis de las relaciones tróficas en un lago de inundación de la Amazonia colombiana. Rev Colombiana Cienc Anim. 2012;4(1):102-120

Andramunio-Acero C. 2013. Dinámica sucesional y ecología trófica de la comunidad perifítica en dos ambientes del sistema lagunar de Yahuarcaca (Amazonas, Colombia). Tesis de grado. Universidad Nacional de Colombia-sede Amazonía. Leticia, Amazonas. $87 \mathrm{p}$

Carney HJ. Productivity, population growth and physiological responses to nutrient enrichments by phytoplankton of Lake Titicaca, Peru-Bolivia. Verh Internat Verein Limnol. 1984;22:1253-1257

Carney HJ, Richerson PJ, Eloranta P. Lake Titicaca (PerulBolivia) phytoplankton: Species composition and structural comparison with other tropical and temperate lakes. Arch Hydrobiol. 1987; 110:365-385

CORPONARIÑO. 2014. Plan de Manejo integral del Humedal Ramsar Laguna de la Cocha. Corporación Autónoma Regional de Nariño. Pasto, Colombia.

CORPONARIÑO. Resolución 1006 de noviembre 09 de 2015. Corporación Autónoma Regional de Nariño. Pasto, Colombia.

Corrales LC, Antolinez DM, Bohórquez JA, Corredor AM. 2015. Anaerobic bacteria: processes they perform and their contribution to life sustainability on the planet. Universidad Colegio Mayor de Cundinamarca. Bogotá, Colombia.

Ding Y, Liu Y, Wu W, Shi D, Yang M, Zhong Z. Evaluation of biochar effects on nitrogen retention and leaching in multi-layered soil columns. Water Air Soil Poll. 2010;213:47-55. Doi: 10.1007/ s11270-010-0366-4

Elser JJ, Marzolf ER, Goldman CR. Phosphorus and nitrogen limitation of phytoplankton growth in freshwaters of North America: a review and critique of experimental enrichments. Can J Fish and Aquat Sci. 1990;47:1468-1477
Gilmore SR, Abbott CL, Cone DK. The placement of Gyrodactylus salmonis (Yin \& Sproston) in the molecular phylogeny of studied members of the Gyrodactylus wageneri group parasitizing salmonids. J Fish Dis. 2010;33:461-467

Granados C, Rodríguez C, Mancera JC, López HJ. 2008. Producción primaria fitoplanctónica, respiración, balance metabólico y relación con variables fisicoquímicas durante la época lluviosa de 2007 en la Ciénaga Grande de Santa Marta, Caribe colombiano. Resúmenes VIII Seminario Colombiano de Limnología. Santiago de Cali. pp. 102.

Hakspiel C. 2005. Variación espacial y temporal de la abundancia de nanoplancton heterótrofofo y su relación con la abundancia y volumen celular del bacterioplancton en el Embalse del Neusa. Facultad de ciencias naturales. Universidad Jorge Tadeo Lozano. Bogotá. 99p

Hela I, Laveastu T. The influence of temperature on the behavior of fish. Arch Soc Zool Bot Fenn Vanamo. 1952;15:1-2

Kirk J. 1994. Light and photosynthesis in aquatic ecosystems. Cambridge University Press, London, 509 pp.

Khalida A, Arshadb M, Anjuma M, Mahmooda T, Dawsonc L. The anaerobic digestion of solid organic waste. Elsevier [revista en internet]* 2011 [acceso 10 Marzo 2014]; 31(8). Disponible en: http://www.researchgate.net/publication/51086548.The_ anaerobic_digestion_of_solid_organic_waste.

Kormondy E. 1994. Conceptos de Ecología Aplicada. Madrid, España: Alianza Editorial

López J, Mena P, Torres C, Rodríguez J, Imuez M, Burgos A. 2005. Evaluación de inmunoestimulantes en las fases de levante y ceba de trucha arcoiris (O. mykiss) cultivada en jaulas flotantes en el Lago Guamuéz. Vicerrectoria de Investigaciones Postgrados y Relaciones Internacionales. Pasto. Colombia: Sistema Investigaciones. p. 6

López-Macías JN. 2014. Nutrición y Alimentación Piscícola. Colombia: Editorial Universidad de Nariño

López-Macias JN, Salas-Benavides J. Caracterización Comparativa de la condición limnológica del lago Guamuéz en relación con la producción de trucha arcoíris Oncorrynchus mykiss en Jaulas Flotantes. Entornos. 2013;26(2): 181-194

Ordoñez YM, Fernandez BR, Lara LS, Rodriguez A, Uribe D, Sanders IR. Bacteria with phosphate solubilizing capacity alter mycorrhizal fungal growth both inside and outside the root and in the presence of native microbial communities. PLoS One. 2016;11(6):e0154438. Doi: 10.1371/journal.pone.0154438

Rábago-Castro JL. 2010. Monitoreo y distribución de infecciones bacterianas y parasitarias en el cultivo de bagre Ictalurus punctatus en Tamaulipas. Tesis de doctorado, Facultad de Ciencias Biológicas, Universidad Autónoma de Nuevo León, Monterrey

Roldán G. 1992. Fundamentos de Limnología Neotropical. Editorial Universidad de Antioquia, Medellín. pp.529

Roldan P. 2001. Estudio limnológico de los recursos hídricos del Parque de Piedras Blancas. Academia Colombiana de Ciencias Exactas, Físicas y Naturales. Bogotá. Pp.137 
Roldán-Pérez G. 2003. Bioindicación de la calidad del agua en Colombia. Antioquia: Universidad de Antioquia

Rubio-Godoy M, Paladini G, Freeman MA, García-Vásquez A, Shinn AP. Morphological and molecular characterisation of Gyrodactylus salmonis (Platyhelminthes, Monogenea) isolates collected in Mexico from rainbow trout (Oncorhynchus mykiss Walbaum). Vet Parasitol. 2012;186:289-300

Sánchez R, Zea S. "Metabolismo de nitrógeno y fósforo inorgánico disueltos en la columna de agua en una laguna costera tropical (Caribe Colombiano)". Caribbean Journal of Science. 2000;36:127-140

Sánchez de Prager M, Rojas A, Pérez J, Zúñiga O, Gascó JM. Actividad y biomasa microbianas como indicadores de materia orgáni- ca en sistemas de cultivo de maracuyá (Passiflora edulis) en Toro, Valle del Cauca, Colombia. Acta Agron. 2006;55(4):7-12

Smith VH. Nutrient dependen of primary productivity in lakes. Limnol Oceanogr. 1983;28:1051-1064

Valdés y Plaza 2011. Organismos indicadores de la calidad del agua y de la contaminación (bioindicadores). (2. ed, Ed.) USA: ProQuest Ebook Central.

Varma A. 2017. (Ed) Soil biology. Springer. New york, USA.

Vincent WF, Wurtsbaugh WA, Vincent CL, Richerson PJ. 1984. Seasonal dynamics of nutrient limitation in a tropical high-aititude lake (Lake Titicaca, Peru-Bolivia): application of Physiological bioassays. Limnol. Oceanogr. 29: 540-552.

Jorge López: https://orcid.org/0000-0001-9571-5524 Julbrinner Salas: https://orcid.org/0000 -0002-0536-8774 